\title{
On the Synthesis Problems of the Semimodular State Chart Theory
}

\section{Distributive Chart}

\author{
By \\ Tsuyoshi NAKAMURA*
}

\begin{abstract}
Introduction
We normally classify switching circuits as either synchronous or asynchronous depending on whether or not the signals in the circuit are synchronized with some source of fundamental frequency (or clock) which regulates the entire circuit. It is possible to predict the state of a synchronous circuit for any given clock signal if one knows the initial state of the circuit and its logical characteristics. However from knowledge of the logical characteristics of the circuit alone, it is impossible in an asynchronous circuit to predict the next state from the present one. The state may also depend upon the relative speeds of some of the logical elements which comprise the circuit. One of the main objectives of asynchronous theory is to describe the properties of circuits in which their ultimate behavior does not depend on the relative speeds of their elements.

The semimodular circuit theory introduced by D. E. Muller and W. S. Bartkey had the purpose of developing the techniques for designing asynchronous circuits $[1,2,3]$. In a series of papers $[4,5,6]$, it was reorganized as a theory of $n$-dimensional space of lattice points. In this theory a state of a circuit is specified by a $n$-tuple $z=\left(z_{1}, \ldots, z_{n}\right), z_{i} \in\{0,1, \ldots, p\}$, and from a sequence of states $z^{1} \rightarrow z^{2} \rightarrow z^{3} \rightarrow \cdots$, we can construct the sequence of $n$-tuples $M^{1} \rightarrow M^{2} \rightarrow$ $M^{3} \rightarrow \cdots$, such that the $i$-th component $M_{i}^{k}$ of $M^{k}=\left(M_{1}^{k}, M_{2}^{k}, \ldots, M_{n}^{k}\right)$, is a nonnegative integer representing the total number of changes of $i$-th component of $z_{i}^{1} \rightarrow z_{i}^{2} \rightarrow z_{i}^{3} \rightarrow \cdots \rightarrow z_{i}^{k}$ during the state change from $z^{1}$ to $z^{k}$. For instance, if $z^{1}=(10), z^{2}=(00), z^{3}=(01), z^{4}=(11), z^{5}=(10), \ldots$, then $M^{1}=(00), M^{2}=(10)$,
\end{abstract}

Communicated by S. Takasu, April 24, 1979.

* School of Medicine, Nagasaki University, Nagasaki 852, Japan. 
$M^{3}=(11), M^{4}=(21), M^{5}=(22), \ldots$ With respect to a preassigned sequence $z^{1} \rightarrow z^{2} \rightarrow z^{3} \rightarrow \cdots,\left(M^{1} \rightarrow M^{2} \rightarrow M^{3} \rightarrow \cdots\right)$, we can define the mapping $h$ of the sequence $\left\{M^{k}\right\}$ into the $n$-dimensional cube as $h\left(M^{k}\right)=z^{k}$. If the circuit satisfies a certain condition, i.e., semimodularity, this $h$ can be a mapping from a subset of the $n$-dimensional lattice point space to the $n$-dimensional cube, where the image $h\left(M^{k}\right)$ of $M^{k}$ does not depend on the sequences connecting the origin $(0, \ldots, 0)$ to $\left(M_{1}^{k}, \ldots, M_{n}^{k}\right)$. Moreover the set of points, which appear in the sequence $\left\{M^{k}\right\}$ induced by a sequence $\left\{z^{k}\right\}$ starting from the preassigned initial state, makes a semimodular lattice in the $n$-dimensional lattice point space. Thus, if the circuits $C$ is semimodular, $C$ determines a pair $(V, h)$ where $V$ is a semimodular lattice and $h$ is a mapping of $V$ into the $n$-dimensional cube, and conversely this $(V, h)$ completely determines the circuit. However, if a pair $(V, h)$ is given arbitrarily, it does not always determine a circuit. To surmount this difficulty, we have to resort to a so called "digital extension" $\left(V^{e}, h^{e}\right)$ of $(V, h)$ so that $\left(V^{e}, h^{e}\right)$ determines a circuit. Such construction is called "synthesis procedure" [3]. Synthesis procedure for distributive charts was formerly treated in $[3,8]$, and that for semimodular charts was first treated in [9]. The purpose of this paper is to describe a simpler synthesis procedure than that of [8] for distributive charts by using the approach which was left incomplete in [3]. We shall briefly discuss on this point at the beginning of Chapter II.

This paper is based on an extensive examination of a finite dimensional lattice point space, especially of its recurrence property. To make the paper easy and self-contained, semimodular state chart theory is briefly reviewed with intuitive examples, and discussions are limited on binary state charts because the binary case is mosi practical and other $p$-ary cases, $p \geqq 3$, may be seen from analogies. Subsequent to the original publication of the semimodular theory, there have been considered some mathematical models to evaluate parallelism of computations. The connection of them have been investigated in [15-18]. This paper treats the distributive case; the subsequent paper will treat the semimodular case.

\section{Chapter I. The State Chart}

In this chapter, we shall briefly refer to the symbols, definitions and theorems of semimodular state charts theory with a few new counter examples. For more detailed results, the reader should refer to $[1,2]$ or $[4,5,6]$. 


\section{$\S 1$. The Similarity Relation}

Definition 1.1. We denote the set of all non-negative integers i.e., $\{0,1$, $2, \ldots\}$ by $W . \quad J$ denotes a finite set of indices, usually $J=\{1,2, \ldots, n\}$, unless explicitly defined. The elements of $J$ are called the nodes of $J . W^{J}$ denotes the cartesian product whose element $M$ is a $n$-dimensional line vector $\left(M_{1}, \ldots\right.$, $M_{n}$ ) where $n$ is the number of integers contained in $J$. Let $M$ and $N$ be points in $W^{J}$. The expressions $M \leqq N$ and $M=N$ are defined as $M_{j} \leqq N_{j}$ and $M_{J}=N_{j}$ respectively for each $j$ of $J . \quad W^{J}$ is a partially ordered set with respect to $\leqq$. $M \leqq N$ but $M \neq N$ is denoted by $M<N$. The expressions $M+N, M-N$ (if $M \geqq N), M \vee N$ and $M \wedge N$ represent the points in $W^{J}$ defined by:

$$
\begin{aligned}
& (M+N)_{j}=M_{j}+N_{j} \\
& (M-N)_{j}=M_{j}-N_{j} \\
& (M \vee N)_{j}=\max \left(M_{j}, N_{j}\right) \\
& (M \wedge N)_{j}=\min \left(M_{j}, N_{j}\right)
\end{aligned}
$$

for all $j$ of $J$ respectively. We denote $\delta^{i}$ and $0^{J}$ as points in $W^{J}$ such that $\left(\delta^{i}\right)_{j}=\delta_{i j}$ (the Kronecker delta), and $\left(0^{J}\right)_{j}=0$ for each $j$ of $J$. Let $Y$ be a subset of $W^{J}$. A point $M$ in $Y$ is minimal in $Y$ if there is no other point $L$ in $Y$ such that $L<M$. We say that $N$ covers $M$ in $Y$ if $N, M$ are in $Y, N>M$, and there is no other point $L$ in $Y$ such that $N>L>M$.

Lemma 1.2. In any subset $Y$ of $W^{J}$ the number of minimal points is finite.

Definition 1.3. A subset $V$ of $W^{J}$ is said to be semimodular if the following conditions are satisfied:

(1) if $M$ and $N$ are in $V$, then $M \vee N$ is in $V$,

(2) if $N$ covers $M$ in $V$, then $N=M+\delta^{i}$ for one $i$ of $J$,

(3) $0^{J}$ is in $V$.

The condition (1) may be changed to (1)*

(1) if $L, L+\delta^{i}$ and $L+\delta^{j}$ are in $V$ and $i \neq j$, then $L+\delta^{i}+\delta^{j}$ is also in $V$. Furthermore if $V$ satisfies condition (4), then $V$ is said to be distributive,

(4) if $M$ and $N$ are in $V$ then $M \wedge N$ is also in $V$.

The condition (4) may be changed to (4)*:

$(4)^{*}$ if $L, L-\delta^{i}$ and $L-\delta^{j}$ are in $V$ and $i \neq j$, then $L-\delta^{i}-\delta^{j}$ is also in $V$. 
Lemma 1.4. Let $V$ be a distributive subset of $W^{J}$. If the set $\{M ; M \in V$ and $\left.M_{i}=\theta\right\}$ is not empty for a node $i \in J$ and a positive integer $\theta$, then it has a unique minimum $P$, hereafter denoted by $P \leftrightarrow[\theta, i]$. Then $P \leqq L$ holds for any point $L \in V$ such that $L_{i} \geqq \theta$.

Lemma 1.5. If $P \leftrightarrow[\theta, i]$ and $P \leftrightarrow[\phi, j]$, then $i=j$ and hence $\theta=\phi$.

Proof. Since $P>0^{J}$, we have a sequence $\{M(k)\}, k=0, \ldots, m$, such that $M(0)=0^{J}, M(m)=P$ and $M(k+1)=M(k)+\delta^{h(k)}$ for $h(k) \in J . \quad$ Let $k_{0}=\max \{k$; $h(k)=i$ or $h(k)=j\}$. Obviously, $k_{0} \leqq m-1$. Assuming $h\left(k_{0}\right) \neq j$, we get $h\left(k_{0}\right)$ $=i$ and $P_{j}=M\left(k_{0}\right)_{j}$. Since $P \leftrightarrow[\phi, j]=\left[M\left(k_{0}\right)_{j}, j\right], P \leqq M\left(k_{0}\right)$. On the other hand $P \geqq M\left(k_{0}+1\right)=M\left(k_{0}\right)+\delta^{i}$, which is a contradiction to the previous result based on the assumption. Therefore $h\left(k_{0}\right)=j$ must hold. Similarly $h\left(k_{0}\right)=i$, hence $i=j$.

Definition 1.6. Let $\{0,1\}^{J}$ denote the set of mappings from $J$ to $\{0,1\}$. Let $V$ be a semimodular subset of $W^{J}$, and $h: V \rightarrow\{0,1\}^{J}$ be a mapping satisfying the following conditions:

$$
\begin{aligned}
& \text { if } M_{j}=N_{j}, \text { then } h(M)_{j}=h(N)_{j}, \\
& \text { if } M_{j}=N_{j}+1, \text { then } h(M)_{j} \neq h(N)_{j} .
\end{aligned}
$$

As we shall deal with the binary case in this paper, $h$ is defined as a mapping to $\{0,1\}^{J}$. In general, $h$ shall be defined as a mapping to $\{0,1, \ldots, p-1\}^{J}$ in the case of $p$-ary. In the binary case $h(M)_{j}=h\left(0^{J}\right)_{j}+M_{j}(\bmod 2)$ may be easily verified from 1.6. Thus, it should be noted that all $h(M)$ are determined if $h\left(0^{J}\right)$ is specified. The pair $(V, h)$ of $V$ and such a mapping $h$ is called state chart.

As briefly stated in the introduction, given a circuit, we get a set $V$ by accumulating the number of signal changes performed in the circuit and $h(M)$ so as to represent the exact state of the circuit for each $M \in V$. When a circuit satisfies a condition so-called "semimodularity", $V$ is proved to be a semimodular subset and $h$ is as a mapping from $V$ to $\{0,1\}^{J}$ satisfying the condition of 1.6. Thus, the order of signal changes in a semimodular circuit can be completely represented by a semimodular state chart $(V, h)$. In this case, the chart $(V, h)$ satisfies the condition of "digitalness" (refer to 1.21). Conversely, when a real circuit is designed, the order of signal changes is given as a condition. That is, a semimodular state chart $(V, h)$ is given first, but it does not necessarily satisfy the condition of "digitalness". 
Definition 1.7. We define the set $V_{M}=\{N-M ; N \geqq M$ and $N \in V\}$ for each $M$ of $V$. If $V$ is semimodular or distributive then $V_{M}$ is semimodular or distributive respectively.

Definition 1.8. Let $(V, h)$ be a state chart. When $V_{M}=V_{N}$ and $h(M)$ $=h(N)$, two points $M$ and $N$ are called similar, written as $M \sim N$. The similarity relation is an equivalence relation in $V$. The equivalence classes with the similarity relation are called the similarity classes in $(V, h)$, written as $(V, h) / \sim$ We write $T \mathscr{F} S$ for similarity classes $T$ and $S$ if $M \leqq N$ holds for some $M \in T$ and $N \in S$.

The set $V_{M}$ tells us the order of signal changes from the state $h(M)$ of a circuit. Therefore, if $(V, h)$ represents the order of signal changes of a real circuit, $V_{M}=V_{N}$ should hold whenever $h(M)=h(N)$. However, since $(V, h)$ does not necessarily represent a real circuit, $h(M)=h(N)$ does not imply $V_{M}=V_{N}$. In Figure 2, (200) (020) but $(200)+(220)$.

Lemma 1.9. Let $(V, h)$ be a state chart. If $M \sim N$ and $L \in V_{M}$ then $M+L \sim N+L$.

Proof. Let $P \in V_{L+M}$, then $P+L \in V_{M}, P+L \in V_{N}$ and $P \in V_{L+N}$, therefore $V_{L+M} \subset V_{L+N}$. Similarly, $V_{L+N} \subset V_{L+M}$. Since $h(M)=h(N), M_{j} \equiv N_{j}(\bmod 2)$ for each $j \in J$, hence $(M+L)_{j} \equiv(N+L)_{j}(\bmod 2)$ for each $j \in J$ and therefore $h(M+L)=h(N+L)$.

Lemma 1.10. Let $(V, h)$ be a state chart and $M, N$ be points in $V$ such that $M \sim N$. Then $V_{M} \subset V_{M \vee N}$ and $h(M)=h(M \vee N)$.

Proof. Let $L \in V_{M}=V_{N}$, then both $M+L$ and $N+L$ are in $V$, therefore $(M+L) \vee(N+L)=(M \vee N)+L \in V$ and $L \in V_{M \vee N}$. Since $h(M)=h(N), h(M \vee N)$ $=h(M)$.

Theorem 1.11. Let $(V, h)$ be a distributive chart and $M$ and $N$ be points in $V$ such that $M \sim N$. Then $M \sim N \sim M \vee N \sim M \wedge N$.

Proof. This follows from ordinary calculations.

\section{§2. The Cycles}

Definition 1.12. Let $(V, h)$ be a state chart with nodes $J$. For a point $M$ of $V$ we define $Z(M)$ as a set of non-zero points $Z$ of $W^{J}$ where $M+Z$ covers $M$ 
in the similarity class containing $M$. The elements of $Z(M)$ are called the cycles of $Z(M), Z(M)$ can have only a finite number of cycles (1.2). If $Z$ is a cycle then $Z_{j} \equiv 0(\bmod 2)$ for all $j \in J$. By 1.9 , if $M \sim N$ then $Z(M)=Z(N)$. Thus cycles are the same for any point in the same similarity class $T$, which are called the cycles of $T$, written as $Z(T)$.

Lemma 1.13. Let $(V, h)$ be a state chart and a point $M$ be in a similarity class $T$. Then a point $N$ in $T$ such that $N \geqq M$ is written as the form; $N=$ $M+a(1) Z(1)+a(2) Z(2)+\cdots+a(m) Z(m)$ where $a(i) \in W$ and $Z(i) \in Z(T)$. Conversely, a point $N$ written in the above form is a point in $T$.

Proof. The former part is proved by taking covering points from $M$ to $N$ in $T$ one after another. The latter part is a consequence of 1.9.

Corollary 1.14. Let $(V, h)$ be a state chart, $M$ and $N$ be points in $V$ such that $M \leqq N$ and $Z$ be a cycle in $Z(M)$. Then $Z=a(1) X(1)+\cdots+a(m) X(m)$ for the cycles $X(j)$ of $Z(N)$ and $a(j) \in W$.

Theorem 1.15. Let $(V, h)$ be a state chart and $T, S$ be similarity classes such that $T \mathscr{F} S$ and $S \mathscr{F} T$. Then $Z(T)=Z(S)$ holds.

Proof. Let $Z(S)=\{X(1), \ldots, X(n)\}$ and $Z(T)=\{Y(1), \ldots, Y(m)\}$. Since $S \mathscr{F} T$,

$$
Y(i)=a(i, 1) X(1)+\cdots+a(i, n) X(n)
$$

for nonnegative integer $a$ 's. Similarly,

$$
X(j)=b(j, 1) Y(1)+\cdots+b(j, m) Y(m) .
$$

Assigning (2) to (1) and rearranging,

$$
\begin{aligned}
Y(i)=\{a(i, 1) b(1,1) & +\cdots+a(i, n) b(n, 1)\} Y(1)+\cdots \\
\cdots+ & \{a(i, 1) b(1, m)+\cdots+a(i, n) b(n, m)\} Y(m)
\end{aligned}
$$

for each $i . \quad$ Since $Y(i) \geqq Y(j)$ can not hold unless $i=j$,

$$
a(i, 1) b(1, i)+\cdots+a(i, n) b(n, j)= \begin{cases}1 & \text { if } j=i, \\ 0 & \text { otherwise }\end{cases}
$$

Similarly by assigning (1) to (2), we have

$$
b(j, 1) a(1, i)+\cdots+b(j, n) a(n, i)= \begin{cases}1 & \text { if } j=i, \\ 0 & \text { otherwise } .\end{cases}
$$

Let us consider a fixed node $i$. There exists $k$ such that $a(i, k) \neq 0$. Then it 
follows from (3) and (4) that

$$
b(k, j)=0 \quad \text { if } \quad j \neq i
$$

and

$$
b(j, i)=0 \quad \text { if } \quad j \neq k .
$$

Since $1=a(i, 1) b(1, i)+\cdots+a(i, m) b(m, i)=a(i, k) b(k, i)$, we have $a(i, k)=$ $b(k, i)=1$ and consequently $X(k)=b(k, i) Y(i)=Y(i)$. Thus we may find a cycle $X(k)$ for each $Y(i)$ such that $X(k)=Y(i)$, and therefore $Z(T) \supset Z(S)$. Since $Z(S)$ $\supset Z(T)$ also follows from the similar argument, this completes the proof.

Lemma 1.16. Let $(V, h)$ be a distributive chart and $T$ be a similarity class such that $Z(T)$ has two distinct cycles $Z$ and $X$. Then $Z \wedge X=0^{J}$.

Proof. This follows from 1.11 .

Definition 1.17. Let $(V, h)$ be a state chart with nodes $J$ and $Z$ be a cycle of $Z(M)$ for a point $M$ in $V$. We write $s(Z)=\left\{i ; Z_{i} \neq 0\right\}$ and $u(Z)=\left\{i ; Z_{i}=0\right\}$, called spanned nodes and unspanned nodes of $Z$ respectively. It is clear that $s(Z) \cup u(Z)=J$.

Lemma 1.18. Let $(V, h)$ be a distributive chart, $M$ and $N$ be points in $V$ such that $M \leqq N$. Then $Z(M) \subset Z(N)$.

Proof. Let $Z$ be a cycle in $Z(M)$. Then there exists a cycle $X$ in $Z(N)$ such that $Z \geqq X$ (1.14). There exists an integer $m$ such that $(M+m Z)_{i} \geqq N_{i}$ for any $i$ of $s(Z)$. Let $P$ and $Q$ stand for $M+m Z$ and $(M+m Z) \wedge N$ respectively. Since $M \leqq Q \leqq P$ and $M \sim P, Z \in Z(Q)$ (1.15). Since $((P+Z) \wedge(N+X))_{i}=$ $(N+X)_{i}=N_{i}+X_{i}=Q_{i}+X_{i}=(Q+X)_{i}$ for any $i$ of $s(Z),((P+Z) \wedge(N+X))_{i}=P_{i}$ $=Q_{i}=(Q+X)_{i}$ for any $i$ of $u(Z)$, we have $Q+X \in V$ because $P+Z \in V$ and $N+X$ $\in V$. Then it follows from a relatively long but natural calculation that $Q \sim$ $Q+X$. Since $Z \in Z(Q), Z \geqq X$ and $Q \sim Q+X, X$ should be equal to $Z$ and $Z=X \in Z(N)$.

Lemma 1.19. Let $(V, h)$ be a distributive chart and $M, N$ be points in $V$ such that $Z(M)$ and $Z(N)$ have the same cycle $Z$. Then $Z$ is also a cycle in $Z(M \wedge N)$.

Proof. $\quad M \wedge N \sim(M \wedge N)+Z$ follows from a simple calculation using the hypothesis $M \sim M+Z$ and $N \sim N+Z$. Then $Z(M \wedge N)$ should have a cycle $X$ such that $X \leqq Z$. Since $Z$ and $X$ are cycles in $Z(M)(1.18)$ and $X \wedge Z \neq 0^{J}, X$ should be equal to $Z$. 
Theorem 1.20. Let $(V, h)$ be a distributive chart with nodes $J$. If $X$ and $Z$ are cycles of $(V, h)$ which are different, then $Z \wedge X=0^{J}$. For each cycle $Z$, the set $\{M ; Z \in Z(M)\}$ has the unique minimum point, which will be called as the minimum point with respect to the cycle $Z$.

Proof. Let $M$ and $N$ be points in $V$, and $X$ and $Z$ be cycles in $Z(M)$ and $Z(N)$ respectively. Then $X$ and $Z$ are also cycles in $Z(M \wedge N)$ (1.18) and therefore $X \wedge Z=0^{J}$ (1.16). The set $\{M ; Z \in Z(M)\}$ has at most finite number of minimal points $L(1), \ldots, L(m)(1.2)$, then $L(1) \wedge \cdots \wedge L(m)$ also is a point in the set (1.19) and therefore the set has the unique minimum point.

\section{§3. Finiteness and Digitalness}

Definition 1.21. A state chart $(V, h)$ is finite if the number of the similarity classes in $(V, h)$ is finite. A state chart is digital if $M \sim N$ whenever $h(M)=$ $h(N)$. A state chart is orthogonal if $X \wedge Z=0^{J}$ whenever $X$ and $Z$ are cycles of the same similarity class and $X \neq Z$. Distributive charts are orthogonal (1.16). Clearly, digital charts are finite.

The digitalness is a necessary and sufficient condition for a semimodular state chart $(V, h)$ to represent the order of signal changes of a real circuit. When a non-digital state chart $(V, h)$ is given to be realized, we shall try to find a "digital extension" of $(V, h)$ by adding some new nodes and construct a real circuit from the digital extension. Then we ignore the added new nodes and pay attention only to the ordinary nodes, whose signal changes coincide with those specified by $(V, h)$. Finding a digital extension is the synthesis problem. (An algorithm to construct a real circuit from a digital extension is described in $[12,13]$.)

Theorem 1.22. A finite state chart $(V, h)$ is orthogonal.

Proof. Let $X$ and $Y$ be different cycles of a similarity class $T$, and let $M \in T$. Then $M+n(X \vee Y)=(M+n X) \vee(M+n Y) \in V$ for any $n \geqq 0$. Since $(V, h)$ is finite, $M+n(X \vee Y) \sim M+m(X \vee Y)$ for some $m>n$. On the other hand, since

$$
V_{M+n(X \vee Y)} \subset V_{M+n(X \vee Y)+(X \vee Y)} \subset \cdots \subset V_{M+m(X \vee Y)}
$$

by 1.10 , we have $V_{M+n(X \vee Y)}=V_{M+(n+1)(X \vee Y)}$. Since $X_{j} \equiv Y_{j} \equiv 0(\bmod 2)$, $h(M+n(X \vee Y))=h(M+(n+1)(X \vee Y))$, hence $M+n(X \vee Y) \sim M+(n+1)(X \vee$ $Y)$. Then, by $1.15, M+n(X \vee Y) \sim M+n(X \vee Y)+X \sim M+n(X \vee Y)+(X \vee Y)$ 
$\sim M+n(X \vee Y)+X+Y$. Since $Y$ is a cycle of $Z(M+n(X \vee Y)+X)$ by 1.15 , $X \vee Y=X+Y$ should hold. Then we have $X \wedge Y=X+Y-(X \vee Y)=0^{J}$.

\section{§4. Extension and Amalgamation}

The dimension of a state chart corresponds to the number of elements contained in a circuit. When some new elements are added to the circuit, then we obtain a higher dimensional state chart than the first one. Thus, if a given state chart is not digital, then we may try to find a higher dimensional digital state chart of which restriction coincides with the given one. This situation is expressed as follows.

Definition 1.23. Let $J, J^{e}$ be non-empty finite set of indices such that $J^{e} \supset J$. For a point $M^{e}$ of $W^{j}$, we define a point $M^{e} \mid J$ of $W^{J}$ as $\left(M^{e} \mid J\right)_{j}=$ $M_{j}^{e}$ for each $j$ of $J$. For a subset $V^{e}$ of $W^{J^{e}}$, we define the subset $V^{e} \mid J$ of $W^{J}$ as $V^{e}\left|J=\left\{M^{e} \mid J ; M^{e} \in V^{e}\right\} . \quad V^{e}\right| J$ and $M^{e} \mid J$ are called the grounds of $V^{e}$ and $M^{e}$ in $J$ respectively, which in turn, are extensions of $V^{e} \mid J$ and $M^{e} \mid J$ onto $J^{e}$ respectively. If $V^{e}$ is semimodular or distributive, then $V^{e} \mid J$ is also semimodular or distributive respectively. Correspondingly, for a mapping $h^{e}$ from $V^{e}$ to $\{0,1\}^{J^{e}}$, we define the mapping $h^{e} \mid J$ from $V^{e} \mid J$ to $\{0,1\}^{J}$ as follows: For a point $M \in V^{e} \mid J,\left(\left(h^{e} \mid J\right)(M)\right)_{j}=h^{e}\left(M^{e}\right)_{j}$ for all $j$ of $J$ where $M^{e}$ is any point in $V^{e}$ such that $M^{e} \mid J=M$. Given a state chart $\left(V^{e}, h^{e}\right)$ with nodes $J^{e}$ and $J \subset J^{e},\left(V^{e}\left|J, h^{e}\right| J\right)$ is a state chart with nodes $J$, which will be written by $\left(V^{e}, h^{e}\right) \mid J$ and called the ground of $\left(V^{e}, h^{e}\right)$ in $J$ or conversely, $\left(V^{e}, h^{e}\right)$ is an extension of $(V, h)$ onto $J^{e}$.

Lemma 1.24. Let $(V, h)$ be an extension of a state chart $(V, h)$ with nodes $J$. If $M^{e} \sim N^{e}$ in $\left(V^{e}, h^{e}\right)$ then $M^{e}\left|J \sim N^{e}\right| J$. Therefore $(V, h)$ is finite whenever $\left(V^{e}, h^{e}\right)$ is finite.

Proof. This immediately follows from the definition.

Definition 1.25. Let $J^{k}$ be a finite non-empty set of indices where $k$ ranges over a finite non-empty set $K$. The system $\left\{J^{k}\right\}, k \in K$, is co-intersectional if $J^{r} \cap J^{m}$ are always the same set $J$ for any distinct $r$ and $m$ in $K$. Let $\left(V^{k}, h^{k}\right)$ be state charts with nodes $J^{k}, k \in K$. The system of state charts $\left\{\left(V^{k}, h^{k}\right)\right\}$ is co-intersectional when $\left\{J^{k}\right\}$ is co-intersectional and $\left(V^{m}, h^{m}\right)\left|J=\left(V^{r}, h^{r}\right)\right| J$ for all $r$ and $m$ where $J=\cap J^{k}, k \in K$. Let $J^{e}=\cup J^{k}, k \in K$. We define the 
subset $\otimes V^{k}, k \in K$, of $W^{J^{e}}$ by $\otimes V^{k}=\left\{M^{e} \in W^{J^{e}} ; M^{e} \mid J^{k} \in V^{k}\right.$ for all $\left.k \in K\right\}$, called the amalgamation of $V^{k}, k \in K$. It is noted that if $J$ is empty then the amalgamation is equal to the cartesian product. If each $V^{k}$ is semimodular or distributive, then $\otimes V^{k}$ is also semimodular or distributive respectively. Correspondingly, we define the mapping $\otimes h^{k}, k \in K$, from $\otimes V^{k}$ to $\{0,1\}^{J^{e}}$ as follows: For a point $M^{e} \in \otimes V^{k},\left(\otimes h^{k}\left(M^{e}\right)\right)_{j}=h^{k}\left(M^{e} \mid J^{k}\right)_{j}$ if $j \in J^{k}$. Then $\left(\otimes V^{k}, \otimes h^{k}\right)$ is a state chart with nodes $J^{e}$, which will be written as $\otimes\left(V^{k}, h^{k}\right)$, $k \in K$, and called the amalgamation of $\left(V^{k}, h^{k}\right)$ 's. $\otimes\left(V^{k}, h^{k}\right)$ is an extension of $\left(V^{k}, h^{k}\right)$ for each $k \in K$.

Theorem 1.26. Let $\left\{\left(V^{k}, h^{k}\right)\right\}, k \in K$, be a co-intersectional system of state charts with nodes $\left\{J^{k}\right\}$. If $M^{e}$ and $N^{e}$ are points in $\otimes V^{k}, k \in K$, then $M^{e} \sim$ $N^{e}$ if and only if $M^{e}\left|J^{k} \sim N^{e}\right| J^{k}$ for all $k \in K$.

Proof. Let $V^{e}$ and $h^{e}$ stand for $\otimes V^{k}$ and $\otimes h^{k}$ respectively. Since $\left(V^{e}, h^{e}\right)$ is an extension of $\left(V^{k}, h^{k}\right)$ for any $k \in K$, "only if part" follows from 1.24. Assume that $M^{e}$ and $N^{e}$ are points in $V^{e}$ such that $M^{e}\left|J^{k} \sim N^{e}\right| J^{k}$ for all $k \in K$. Evidently $h^{e}\left(M^{e}\right)=h^{e}\left(N^{e}\right)$. Suppose that $L^{e}+M^{e} \in V^{e}$. Then $\left(L^{e}+M^{e}\right) \mid J^{k}=\left(L^{e} \mid J^{k}\right)+\left(M^{e} \mid J^{k}\right) \in V^{k}$ for all $k \in K$. Since $M^{e}\left|J^{k} \sim N^{e}\right| J^{k}$, $\left(L^{e} \mid J^{k}\right)+\left(N^{e} \mid J^{k}\right)=\left(L^{e}+N^{e}\right) \mid J^{k} \in V^{k}$ for all $k \in K$. Therefore $L^{e}+N^{e} \in V^{e}$. Thus we have $V_{M^{e}}^{e} \subset V_{N^{e}}^{e}$. Similarly $V_{N^{e}}^{e} \subset V_{M^{e}}^{e}$ and $M^{e} \sim N^{e}$ are proved.

Lemma 1.27. Let $\left\{\left(V^{k}, h^{k}\right)\right\}, k \in K$, be a co-intersectional system of state charts. If each $\left(V^{k}, h^{k}\right)$ is finite or digital then $\otimes\left(V^{k}, h^{k}\right)$ is finite or digital, respectively.

Proof. This follows from 1.26.

Synthesis procedures are procedures to construct digital extensions of given state charts. A given state chart is, so to speak, an original plan or an outline of a circuit and a digital extension of it is a complete specification for a logical design of the circuit.

\section{§5. The v-Similarity Relation}

Definition 1.28. Let $(V, h)$ be a state chart and $M, N$ be points in $V$. We write $M \stackrel{v}{\sim} N$, read $v$-similar, if $M \sim N \sim M \vee N$. If $(V, h)$ is digital or distributive then $\sim$ and $\stackrel{v}{\sim}$ are equal.

Lemma 1.29. Let $(V, h)$ be an orthogonal state chart, then the v-similarity 
relation is an equivalence relation.

Proof. This follows from 1.13 and 1.15 .

By 1.22 , the $v$-similarity relation in a finite state chart is an equivalence relation. The orthogonality is essential in the previous lemma. To see this,

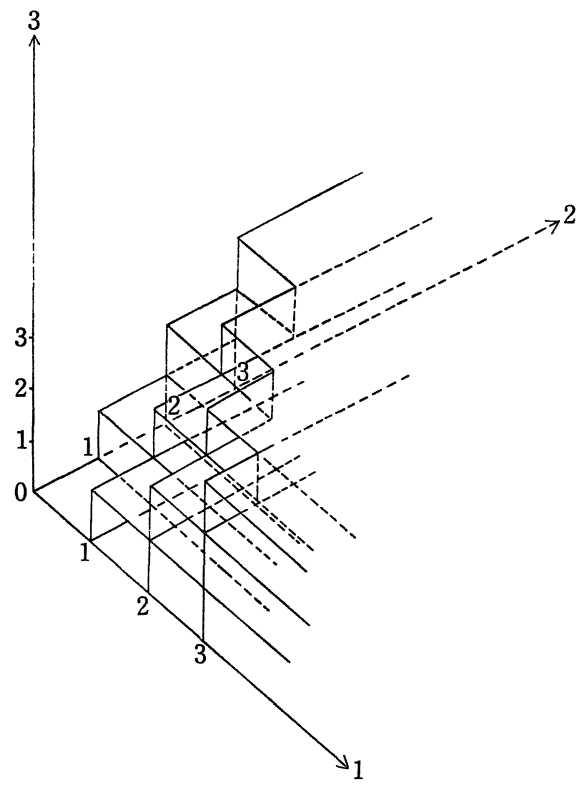

Figure 1. A binary non-orthogonal state chart $(V, h)$ with $V=\left\{\left(x_{1}, x_{2}, x_{3}\right)\right.$ $\left.\in W \times W \times W ; x_{1}+x_{2} \geqq x_{3}\right\}$ and $h(x)_{i}=x_{i}(\bmod 2)$.

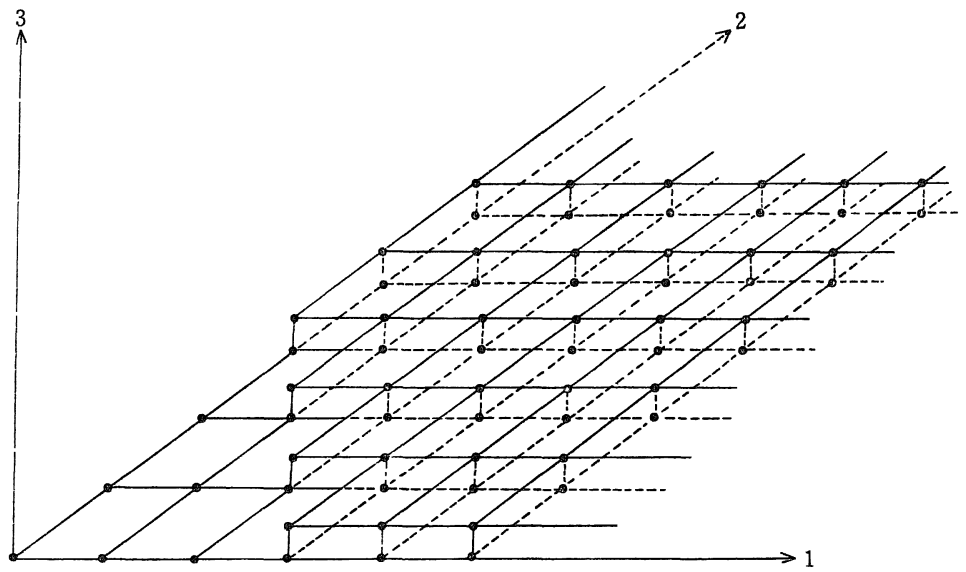

Figure 2. A binary finite state chart $(V, h)$ with $V=\left\{\left(x_{1}, x_{2}, 0\right) ;\left(x_{1}, x_{2}\right) \in W \times W\right\}$ $\cup\left\{\left(x_{1}, x_{2}, 1\right) ; x_{1}+x_{2} \geqq 3\right\}$ and $h(x)_{i}=x_{i}(\bmod 2)$. 
examine the binary state chart of Figure 1 . Though $(000) \stackrel{v}{\sim}(202)$ and $(000) \stackrel{v}{\sim}$ (022), (202) and (022) are not $v$-similar because $(000) \nsucc(222)$. The relations $\sim$ and $\stackrel{v}{\sim}$ are not necessarily equal even in finite siate charts. In the binary finite state chart of Figure 2, (200) (002) but they are not $v$-similar.

Lemma 1.30 (Teorem 2 of [9]). Let $(V, h)$ be a finite state chart with nodes $J$. Then the followings hold.

(1) The number of v-similarity classes in $(V, h)$ is finite.

(2) Let $T$ be a $v$-similarity class and $Z$ be a cycle in $Z(T)$ and $Q$ be the set of nodes spanned by $Z$. Then the set $\{M \mid Q ; M \in T\}$, hereafter denoted by $T \mid Q$, is a totally ordered set.

(3) Let $M$ and $N$ be points in $V$ so that $Z(M)=Z(N)=\{Z(1), \ldots, Z(m)\}$, and let $Q(q)$ be the nodes spanned by $Z(q)$ for each $q \in\{1, \ldots, m\}$. Then $M \stackrel{v}{\sim} N$ if and only if both $M|Q(q) \equiv N| Q(q)(\bmod Z(q) \mid Q(q))$ for all $q \in\{1, \ldots, m\}$ and $M|Q(0)=N| Q(0)$ hold where $Q(0)=J-\cup_{q} Q(q)$.

\section{§6. $\curvearrowleft$-Extension and Digital Extension}

In this section, we shall review some of the results of [8]. The $\kappa$-extension defined in this section is, so to speak, an extension between a given state chart and a digital extension of it.

Definition 1.31. Let $(V, h)$ be a finite state chart and $\{T(\alpha)\}, \alpha=1, \ldots, m$, be the $v$-similarity classes. Taking two $v$-similarity classes $T(\alpha), T(\beta)$, we write $h(T(\alpha))=h(T(\beta))$ if $h(M)=h(N)$ for any points $M \in T(\alpha)$ and $N \in T(\beta)$, furthermore if $M \sim N$ then $T(\alpha) \sim T(\beta)$. Let $K$ be the set of unordered pairs $(T(\alpha)$, $T(\beta)$ ) where $T(\alpha)$ and $T(\beta)$ are both $v$-similarity classes such that $h(T(\alpha))=$ $h(T(\beta))$ but $T(\alpha) \sim T(\beta)$ does not hold: $K=\{(T(\alpha), T(\beta)) ; h(T(\alpha))=h(T(\beta))$ but $T(\alpha)+T(\beta)\}$. The set $K$ is the synthetic class of $(V, h)$ and the elements of $K$ are the knots of $K$.

Definition 1.32. Let $(V, h)$ be a finite semimodular state chart with nodes $J$ and $T, S$ be mutually disjoint subsets of $V$. The extension $\left(V^{e}, h^{e}\right)$ of $(V, h)$ is called as $(T, S)$-extension if it satisfies the following conditions.

(1) If $M^{e}, N^{e}$ are points of $V^{e}$ such that $h^{e}\left(M^{e}\right)=h^{e}\left(N^{e}\right)$ and $M^{e}\left|J \sim N^{e}\right| J$, then $M^{e} \sim N^{e}$.

(2) If $M^{e}, N^{e}$ are points of $V^{e}$ such that $M^{e}\left|J \in T, N^{e}\right| J \in S$, then $h^{e}\left(M^{e}\right) \neq h^{e}\left(N^{e}\right)$. 
Lemma 1.33. Let $(V, h)$ be a finite semimodular state chart with nodes $J$, and let $K$ be the synthetic class of $(V, h)$. If $\kappa$-extension $\left(V^{\kappa}, h^{\kappa}\right)$ with nodes $J$ exists for each $\kappa$ of $K$, then the amalgamation $\otimes\left(V^{\kappa}, h^{\kappa}\right), \kappa \in K$, is a digital extension of $(V, h)$.

Lemma 1.34. Let $(V, h)$ be a finite state chart with nodes $J$, and $T, S$ be mutually disjoint subsets of $V$ which are represented as unions (not necessarily disjoint) as follows:

$$
\begin{aligned}
& T=T(1) \cup \cdots \cup T(m), \\
& S=S(1) \cup \cdots \cup S(n)
\end{aligned}
$$

where $m, n$ are positive integers. Let $\kappa(t, s)$ denote the pair $(T(t), T(s))$ for each $(t, s) \in\{1, \ldots, m\} \times\{1, \ldots, n\}$. If there exists a $\kappa(t, s)$-extension $\left(V^{(t, s)}\right.$, $\left.h^{(t, s)}\right)$ with nodes $J(t, s)$ for each $(t, s)$, then $\otimes\left(V^{(t, s)}, h^{(t, s)}\right),(t, s) \in\{1, \ldots, m\} \times$ $\{1, \ldots, n\}$, is a $(T, S)-$ extension.

Lemma 1.35. Let $J$ be a non-empty finite set of indices. For any points $P, Z$ of $W^{J}$, we denote the set $\{P+r Z ; r$ ranges over integers such that $P+r Z$ $\left.\in W^{J}\right\}$ by $\{P ; Z\}$. Let $P, P^{*}$ and $Z$ be points of $W^{J}$ such that $Z_{i} \neq 0$ for all $i \in J$ and $P \not \equiv P^{*}(\bmod Z)$ i.e., $P-P^{*} \neq k Z$ for any integer $k$. Then there exists an integer $k$ as follows: if we reprcsent $\{P ; Z\},\left\{P^{*} ; Z\right\}$ as unions of mutually disjoint subsets

$$
\begin{aligned}
& \{P ; Z\}=\{P ; k Z\} \cup\{P+Z ; k Z\} \cup \cdots \cup\{P+(k-1) Z ; k Z\} \\
& \left\{P^{*} ; Z\right\}=\left\{P^{*} ; k Z\right\} \cup\left\{P^{*}+Z ; k Z\right\} \cup \cdots \cup\left\{P^{*}+(k-1) Z ; k Z\right\},
\end{aligned}
$$

then for any given $\{P+t Z ; k Z\},\left\{P^{*}+s Z ; k Z\right\}, 0<t, s<k-1$, there exists a member $i$ of $J$ such that $M_{i} \equiv N_{i}(\bmod k Z)$ for all points $M \in\{P+t Z ; k Z\}$, $N \in\left\{P^{*}+s Z ; k Z\right\}$.

Let $P=(2,0), P^{*}=(0,2)$ and $Z=(2,2)$ then $k$ of the lemma is any integer not less than 3 .

\section{Chapter II. Synthesis of Distributive Charts}

In this chapter, we shall present a synthesis procedure for distributive charts. Synthesis procedures are those to construct digital extensions of given state charts. If digital extensions of given state charts are obtained, then circuits which are specified by the state charts can be designed using a set of fundamental 
logic elements. The reader who wants to know more about this should refer to [12] or [13]. The synthesis problem was first dealt with in [3], however, it was left incomplete. A complete procedure for distributive charts was first given in [8]. Briefly speaking, the work required for constructing a digital extension using the procedure of this chapter is proportional to the dimension number $n$ of $(V, h)$ but that used in [8] is proportional to the second power of $n$. One of the differences between them is that [8] studies the grounds of $(V, h)$ in all the two dimensional spaces, while, the present one studies the grounds of $(V, h)$ in the spaces spanned by cycles. The latter approach was the one that [3] tried and left incomplete. The section 1 gives a fundamental relation between $(V, h)$ and the grounds of $(V, h)$ in the spaces spanned by cycles.

\section{§1. q-Similarity Relation}

Definition 2.1. Let $(V, h)$ be a finite distributive chart with nodes $J$ and $Z(1), \ldots, Z(m)$ be the cycles of $(V, h)$ where $m$ is an integer not greater than the number of nodes in $J$ and $Z(i) \wedge Z(j)=0^{J}$ whenever $i \neq j(1.20) . \quad Q(q)$ denotes the set of nodes spanned by a cycle $Z(q)$ for each $q \in\{1, \ldots, m\}$ and the set of nodes not spanned by any cycle is denoted by $Q(0)$, then we have $J=Q(0) \cup Q(1)$ $\cup \cdots \cup Q(m)$ and $Q(i) \cap Q(j)=\phi$ whenever $i \neq j$. Let $q \in\{0,1, \ldots, m\}$, and $V \mid Q(q)$ be the ground of $V$ in $Q(q)$. Two points $A^{q}$ and $B^{q}$ in $V \mid Q(q)$ are $q$-similar, written as $\stackrel{q}{\sim}$, when there exist points $A$ and $B$ in $V$ such that $A\left|Q(q)=A^{q}, B\right| Q(q)=B^{q}$ and $A \sim B$. Obviously $\stackrel{q}{\sim}$ and $=$ are equal to each other when $q=0$.

Lemma 2.2. Let $(V, h)$ be a distributive chart with nodes $J$, and $Z(q)$ and $Q(q)$ be the cycle and the nodes of an element $q \in\{1, \ldots, m\}$ in 2.1. Then two points $A^{q}$ and $B^{q}$ in $V \mid Q(q)$ are $q$-similar whenever $A^{q} \geqq L\left|Q(q), B^{q} \geqq L\right| Q(q)$ and $A^{q} \sim B^{q}$ in $(V, h) \mid Q(q)$ where $L$ is the minimum point with respect to the cycle $Z(q)$.

Proof. Let $Z$ and $Q$ stand for $Z(q)$ and $Q(q)$, respectively. It is no loss of generality that we assume $J=\{1, \ldots, r, \ldots, n\}$ and $Q=\{r+1, \ldots, n\}$. Let $A^{q}$ and $B^{q}$ be points in $V \mid Q$ such that $A^{q} \geqq L\left|Q, B^{q} \geqq L\right| Q$ and $A^{q} \sim B^{q}$. Let $A(i)$, $B(i)$ denote the points in $V$ such that $A(i) \leftrightarrow\left[A_{i}^{q}, i\right], B(i) \leftrightarrow\left[B_{i}^{q}, i\right]$ in $(V, h)$ for each $i \in Q$ (for $\leftrightarrow$, refer to 1.4) and let $A^{*}=A(r+1) \vee \cdots \vee A(n) \vee L, B^{*}=B(r+1)$ $\vee \cdots \vee B(n) \vee L$. We have only to show $A^{*}\left|Q=A^{q}, B^{*}\right| Q=B^{q}$. and $A^{*} \sim B^{*}$ 
to prove the lemma.

(a) Let us prove $A^{*} \mid Q=A^{q}$ and $B^{*} \mid Q=B^{q}$. Since $A(j) \leftrightarrow\left[A_{j}^{q}, j\right]$ in $V$, $A(j) \mid Q \leqq A^{q}$. Hence $A(j)_{i} \leqq A_{i}^{q}=A(i)_{i}$ for any $i, j \in Q$ and therefore $A_{i}^{*}=A(i)_{i}$ $=A_{i}^{q}$ for all $i \in Q$. Thus $A^{*} \mid Q=A^{q}$ and similarly $B^{*} \mid Q=B^{q}$.

(b) Let us prove $A^{*}\left|\bar{Q}=B^{*}\right| \bar{Q}$, where $\bar{Q}=\{1, \ldots, r\}$. Since $Z_{i} \neq 0$, there exists an integer $c$ such that $A_{i}^{q} \leqq(L+c Z)_{i}$ for all $i \in Q$. Then we have $A(i) \leqq$ $L+c Z$ for all $i \in Q$ because $A(i) \leftrightarrow\left[A_{i}^{q}, i\right]$. Hence $L \leqq A(r+1) \vee \cdots \vee A(n) \vee L$ $\leqq(L+c Z)$ and therefore $L\left|\bar{Q}=A^{*}\right| \bar{Q}$. Similarly $L\left|\bar{Q}=B^{*}\right| \bar{Q}$ and $A^{*} \mid \bar{Q}=$ $B^{*} \mid \bar{Q}$ are proved.

(c) We shall prove $A^{*} \sim B^{*}$. Since $A^{*}\left|\bar{Q}=B^{*}\right| \bar{Q}$ and $A^{*} \mid Q=A^{q} \sim B^{q}$ $=B^{*} \mid Q, h\left(A^{*}\right)=h\left(B^{*}\right)$. To prove $V_{A^{*}} \subset V_{B^{*}}$, let $M^{*}$ be a point in $V$ such that $M^{*} \geqq A^{*}$ and let $M(i) \leftrightarrow\left[M_{i}^{*}, i\right]$ for each $i \in J$. Then we have $M^{*}=$ $M(1) \vee \cdots \vee M(n)$ by the similar argument in (a). Let $M^{q}$ stand for $M^{*} \mid Q$. Since $M^{q} \geqq A^{q}$ and $A^{q} \sim B^{q}, M^{q}-A^{q}+B^{q} \in V \mid Q$. Let $N(i)$ denote the point in $V$ defined as $N(i) \leftrightarrow\left[\left(M^{q}-A^{q}+B^{q}\right)_{i}, i\right]$ for each $i \in Q$, and $N^{*}$ be the point in $V$ defined by $N^{*}=\left((L+c Z) \vee M^{*}-c Z\right) \vee N(r+1) \vee \cdots \vee N(n)$, where $c$ is a positive integer satisfying $(L+c Z) \mid Q \geqq M^{q}$. Clearly $\left((L+c Z) \vee M^{*}\right)-c Z \in V$ because $L \sim L+c Z$ and $(L+c Z) \vee M^{*} \in V$. Let $r$ be a positive integer such that $M^{q}-A^{q}+B^{q} \leqq(L+r Z) \mid Q$. Since $N(i) \leftrightarrow\left[\left(M^{q}-A^{q}+B^{q}\right)_{i}, i\right], N(i) \leqq L+r Z$ for all $i \in Q$ and hence $(N(r+1) \vee \cdots \vee N(n))|Q \leqq(L+r Z)| Q=L \mid Q$. Then $N^{*} \mid \bar{Q}$ $=\left(\left((L+c Z) \vee M^{*}-c Z\right) \vee N(r+1) \vee \cdots \vee N(n)\right)\left|Q=M^{*}\right| Q=\left(M^{*}-A^{*}+B^{*}\right) \mid Q$ because $M^{*}|\bar{Q} \geqq L| \bar{Q}$ and $A^{*}\left|\bar{Q}=B^{*}\right| \bar{Q}$. Since $(N(r+1) \vee \cdots \vee N(n)) \mid Q=$ $M^{q}-A^{q}+B^{q}$ by the similar argument in (a) and $M^{q}-A^{q}+B^{q} \geqq B^{q} \geqq L \mid Q$, we have $N^{*}\left|Q=\left(\left((L+c Z) \vee M^{*}-c Z\right) \vee N(r+1) \vee \cdots \vee N(n)\right)\right| Q=(L \mid Q) \vee(N(r+1) \vee \cdots \vee$ $N(n))\left|Q=M^{q}-A^{q}+B^{q}=\left(M^{*}-A^{*}+B^{*}\right)\right| Q$. Thus we have $N^{*}=M^{*}-A^{*}$ $+B^{*} \in V$ and therefore $V_{A^{*}} \subset V_{B^{*}}$ is proved. Since $V_{A^{*}} \supset V_{B^{*}}$ is obtained by the similar argument, $V_{A^{*}}=V_{B^{*}}$ is proved.

Lemma 2.3. Let $(V, h)$ be a distributive chart with nodes $J$, and let $Z(q)$ and $Q(q)$ be the cycle and the set of nodes of an element $q \in\{1, \ldots, m\}$, in 2.1 . Then two points $A^{q}$ and $B^{q}$ in $V \mid Q$ are similar whenever $A^{q} \geqq L\left|Q, B^{q} \geqq L\right| Q$ and $A^{q} \equiv B^{q}(\bmod Z \mid Q)$, where $L$ is the minimum point with respect to the cycle $Z$.

Proof. Let $Z, Q$ stand for $Z(q), Q(q)$ respectively. Let $A^{q} \geqq L \mid Q, B^{q}$ $\geqq L \mid Q$ and $A^{q} \equiv B^{q}(\bmod Z \mid Q)$. It is no loss of generality that we assume $B^{q}=A^{q}+c(Z \mid Q)$ for $c \in W$. Let $A$ be a point in $V$ such that $A \mid Q=A^{q}$ and let 
$A^{*}=A \vee L, B^{*}=A^{*}+c Z$. Because $A^{*} \geqq L$, we have $Z \in Z\left(A^{*}\right)$ and therefore $B^{*} \in V$ and $A^{*} \sim B^{*}$. Hence $A^{*}\left|Q \sim B^{*}\right| Q$ (1.24). Since $A^{*}|Q=(A \vee L)| Q=$ $A^{q}$ and $B^{*} \mid Q=A^{q}+c(Z \mid Q)=B^{q}, A^{q} \sim B^{q}$ is proved.

Theorem 2.4. Let $(V, h)$ be a distributive chart with nodes $J$ and $Z(q)$ and $Q(q)$ be the cycle and the nodes of $q \in\{1, \ldots, m\}$ in 2.1. Then the following three conditions are equivalent when $A^{q}$ and $B^{q}$ are points in $V \mid Q(q)$ such that $A^{q} \geqq L \mid Q(q)$ and $B^{q} \geqq L \mid Q(q)$ where $L$ is the minimum point with respect to the cycle $Z(q)$.

(1) $A^{q} \stackrel{q}{\sim} B^{q}$

(2) $A^{q} \sim B^{q}$

(3) $A^{q} \equiv B^{q}(\bmod Z(q) \mid Q(q))$.

Proof. Let $Z, Q$ stand for $Z(q), Q(q)$, respectively. Since (3) $\rightarrow(2)$ and $(2) \rightarrow(1)$ have been proved in 2.3 and 2.2 respectively, we have only to show $(1) \rightarrow(3)$ to prove the theorem. Let $A^{q} \stackrel{q}{\sim} B^{q}$, then there exist $A$ and $B$ in $V$ such that $A\left|Q=A^{q}, B\right| Q=B^{q}$ and $A \sim B$. Since $A \vee B \sim A \sim B$ (1.11), there exist integers $c(i)$ 's and $d(i)$ 's such that $A \vee B=A+\sum c(i) Z(i)$ and $A \vee B=$ $B+\sum d(i) Z(i)$, where $Z(i)$ 's are the cycles of $Z(A)=Z(B)$. Since the cycles are mutually orthogonal, we have $B^{q} \equiv A^{q}(\bmod (Z \mid Q))$ by taking the ground $(A \vee B) \mid Q$.

Lemma 2.5. Let $(V, h)$ be a finite distributive chart with nodes $J$ and let $Z(q)$ and $Q(q)$ be the cycle and the nodes of $q \in\{1, \ldots, m\}$ respectively in 2.1 . Then for any point $A^{q} \in V^{q}$, the set $\left\{M^{q} \in V \mid Q(q) ; M^{q} \geq A^{q}\right\}$ has at most finite points.

Proof. Let $Z$ and $Q$ stand for $Z(q)$ and $Q(q)$, respectively. Supposing that $\left\{M^{q} \in V \mid Q ; M^{q} \geq A^{q}\right\}$ has infinitely many points $\left\{M^{q}(s)\right\}, s=1,2, \ldots$, for some $A^{q} \in V^{q}$, there exist points $\{M(s)\}, s=1,2, \ldots$, in $V$ such that $M(s) \mid Q=M^{q}(s)$ for all $s$. Since $(V, h)$ is finite, there exists an infinite sequence $s(1), s(2), \ldots$ such that $\{M(s(k))\}, k=1,2, \ldots$, are contained in the same similarity class $T$. On the other hand, since $M(s(k)) \mid Q, k=1,2, \ldots$, are mutually distinct, $Z(T)$ has a cycle which spans a node in $Q$ and therefore $Z \in Z(T)(1.20)$. Then we can easily prove that there should exist a point $M(s(k))$ such that $M(s(k)) \mid Q \geqq A^{q}$, because $Z$ spans the nodes of $Q$ and $(M(s(k)) \mid Q)$ 's are mutually distinct. However this contradicts $M(s(k)) \mid Q \geq A^{q}$.

Corollary 2.6. Let $(V, h)$ be a finite distributive chart with nodes $J$ and 
$Z(q)$ and $Q(q)$ be the cycle and the nodes of $q \in\{1, \ldots, m\}$ respectively in 2.1 . Then the number of the equivalence classes of $(V, h) \mid Q$ with respect to $\stackrel{q}{\sim}$ is finite.

Proof. This follows from 2.5 and 2.4 .

\section{§2. $\hat{q}$-Extension}

In this section, $\hat{q}$-similar (a finer relation than the $q$-similar) is defined and the problem of finding digital extensions is reduced to the problem of finding $\hat{q}$-extension.

Definition 2.7. Let $(V, h)$ be a finite distributive chart with nodes $J$ and $Z(q)$ and $Q(q)$ be the cycle and the nodes of $q \in\{1, \ldots, m\}$ respectively in 2.1 . Let $\Phi(q)$ be the subset of $V \mid Q$ defined as $\Phi(q)=\{M \mid Q(q) ; M \geqq L\}$, where $L$ is the minimum point with respect to the cycle $Z(q)$. By $2.5 \Phi(q)$ is a finite set. Let $\Phi(q)$ be $\left\{A^{q}(1), \ldots, A^{q}(m)\right\}$ and $\hat{L}^{q}$ be the point in $V \mid Q$ defined by $\hat{L}^{q}=A^{q}(1)$ $\vee \cdots \vee A^{q}(m)$. It should be noted that if $M \mid Q \geqq \hat{L}^{q}$ then $M \geqq L$ for a point $M \in V$. Two points $A^{q}$ and $B^{q}$ in $V \mid Q$ are $\hat{q}$-similar, written as $A^{q} \stackrel{\hat{q}}{\sim} B^{q}$, when either $A^{q}=B^{q}$ or $\left.A^{q} \ddagger \hat{L}^{q}, B^{q}\right\rfloor \hat{L}^{q}$ and $A^{q} \equiv B^{q}(\bmod Z \mid Q)$. On the other hand, for $q=0$, we define $A^{q} \stackrel{\hat{q}}{\sim} B^{q}$ if $A^{q}=B^{q}$ for points $A^{q}, B^{q}$ in $V \mid Q(0)$. The three relations $A^{q} \stackrel{\hat{q}}{\sim} B^{q}, A^{q} \stackrel{q}{\sim} B^{q}$ and $A^{q} \equiv B^{q}(\bmod Z \mid Q)$ are equal when $A^{q}$ $\pm \hat{L}^{q}$ and $B^{q} \pm \hat{L}^{q}$ by 2.5 .

Lemma 2.8. Let $(V, h)$ be a finite distributive chart with nodes $J$ and let $Z(1), \ldots, Z(m)$ be the cycles of $(V, h)$ which span the nodes $Q(1), \ldots, Q(m)$, respectively, and $Q(0)=J-\cup Q(q)$ as in 2.1. Then two points $A$ and $B$ in $V$ are similar whenever $A \mid Q(q)$ and $B \mid Q(q)$ are $\hat{q}$-similar for all $q \in\{0,1, \ldots, m\}$.

Proof. Let $L(q)$ be the minimum point with respect to the cycle $Z(q)$ and $\hat{L}^{q}$ be the point defined in 2.7 for each $q \in\{1, \ldots, m\}$. Suppose that $A$ and $B$ are points in $V$ such that $A|Q(q) \stackrel{\hat{q}}{\sim} B| Q(q)$. We first prove $A \vee B \sim A$. Since $(A \vee B)|Q(q) \stackrel{\hat{q}}{\sim} A| Q(q)$ holds, there exists a non-negative integer $c(q)$ such that $(A \vee B)|Q(q)=A| Q(q)+c(q)(Z(q) \mid Q(q))$ for each $q \in\{1, \ldots, m\}$. Then we have $A \vee B=A+\sum c(q) Z(q)$ because $(A \vee B)|Q(0)=A| Q(0)$. Thus we only have to show $Z(q) \in Z(A)$ for any $q$ such that $c(q) \neq 0$ to prove $A \vee B \sim A$. Assuming $c(q) \neq 0$ for an integer $q,(A \vee B)|Q(q) \neq A| Q(q)$ and therefore $A \mid Q(q) \ddagger \hat{L}^{q}$, then $A \geqq L(q)$ and $Z(q) \in Z(A)$. Similarly we may prove $A \vee B \sim B$ and therefore $A \sim B$ is proved. 
Definition 2.9. Let $(V, h)$ be a finite distributive chart with nodes $J$ and let $Q(q)$ be the nodes in 2.1 for $q \in\{0,1, \ldots, m\}$. A digital extension $\left(\hat{\nabla}^{q}, \hat{h}^{q}\right)$ of $(V, h) \mid Q(q)$ is called a $\hat{q}$-extension of $((V, h) \mid Q(q))$ when it satisfies the following condition:

(1) If $\hat{h}^{q}\left(\hat{A}^{q}\right)=\hat{h}^{q}\left(\hat{B}^{q}\right)$ for points $\hat{A}^{q}$ and $\hat{B}^{q}$ in $\hat{V}^{q}$, then $A^{q}\left|Q(q) \stackrel{\hat{q}}{\sim} B^{q}\right| Q(q)$.

Theorem 2.10. Let $(V, h)$ be a finite distributive chart with nodes $J$ and let $Q(q)$ 's be the nodes in 2.1 for $q \in\{0,1, \ldots, m\}$. If there exists a $\hat{q}$-extension $\left(\hat{V}^{q} \hat{h}^{q}\right)$ with nodes $J(q)$ for each $q \in\{0,1, \ldots, m\}$ and $J(q)$ 's are mutually disjoint, then the amalgamation $\otimes\left(\left(\hat{\nabla}^{q}, \hat{h}^{q}\right) \otimes(V, h)\right), q \in\{0,1, \ldots, m\}$, is a digital extension of $(V, h)$.

Proof. Let $\left(V^{e}, h^{e}\right)$ stand for the state chart $\otimes\left(\left(\hat{V}^{q}, \hat{h}^{q}\right) \otimes(V, h)\right) . \quad$ In the first place each $\left(\hat{V}^{q}, \hat{h}^{q}\right) \otimes(V, h)$ is an extension of $(V, h)$, and so is $\left(V^{e}, h^{e}\right)$. Assuming $h^{e}\left(M^{e}\right)=h^{e}\left(N^{e}\right)$ for points $M^{e}$ and $N^{e}$ in $V^{e}, \hat{h}^{q}\left(M^{e} \mid J(q)\right)=$ $\hat{h}^{q}\left(N^{e} \mid J(q)\right)$ hold for all $q$. Then we have $M^{e}\left|J(q) \sim N^{e}\right| J(q)$ and $M^{e} \mid Q(q) \stackrel{\hat{q}}{\sim}$ $N^{e} \mid Q(q)$ for all $q$ (2.9). Hence $M^{e}\left|J \sim N^{e}\right| J$ (2.8) and therefore $M^{e}\left|J \cup J(q) \sim N^{e}\right| J \cup J(q)$ in $\left(\widehat{V}^{q}, \hat{h}^{q}\right) \otimes(V, h)$ for all $q(1.26)$. Then it also follows from 1.29 that $M^{e} \sim N^{e}$.

\section{§3. Cumulative Gray Coding}

Definition 2.11. Let $n$ be an integer $\geqq 2$ and $k$ be the integer determined by $k-1<\log _{2} n \leqq k$. We define the mapping $G^{n}:\{0,1, \ldots, n-1\} \rightarrow W^{\Sigma}$ recursively, where $\Sigma$ is a set of $k$ nodes $\{\xi(1), \ldots, \xi(k)\}$, as follows.

1. Let $G^{n}(0)=0^{\Sigma}=(0, \ldots, 0), G^{n}(1)=\delta^{\xi(1)}=(1,0, \ldots, 0)$.

2. Assume that for some $e,(k>e \geqq 1), G^{n}(m),\left(0 \leqq m<2^{e}-1\right)$, have been defined with $G^{n}(m)_{\xi(i)}=0$ for all $i>e$. Then define $G^{n}(m), 2^{e} \leqq m<2^{e+1}$, as follows. For all of these integers $m$, set $G^{n}(m)_{\xi(e+1)}=1$ and set $G^{n}(m)_{\xi}=0$ for all $i>e+1$. For each integer $\left(2^{e}+j\right), 0 \leqq j<2^{e}$, set $G^{n}\left(2^{e}+j\right)_{\xi(i)}=G^{n}\left(2^{e}-1\right)_{\xi(i)}$ $+G^{n}\left(2^{e}-1\right)_{\xi(i)}-G^{n}\left(2^{e}-1-j\right)_{\xi(i)}=2 G^{n}\left(2^{e}-1\right)_{\xi(i)}-G^{n}\left(2^{e}-1-j\right)_{\xi(i)}$ for all $i \leqq e$.

For instance, $G^{5}(0)=(000), G^{5}(1)=(100), G^{5}(2)=(110), G^{5}(3)=(210), G^{5}(4)$ $=(211)$ and $G^{10}(0)=(0000), G^{10}(1)=(1000), G^{10}(2)=(1100), G^{10}(3)=(2100)$, $G^{10}(4)=(2110), G^{10}(5)=(3110), G^{10}(6)=(3210), G^{10}(7)=(4210), G^{10}(8)=(4211)$, $G^{10}(9)=(5211)$. The definition of $G$ may remind us of the reflected Gray code state assignment (refer to 3.3.1 in [14]). We call the mapping $G^{n}$ the cumulative Gray mapping for $n$ with nodes $\Sigma$ or simply Gray mapping for $n$ 
with nodes $\Sigma$. When $n$ is understood, we will denote $G^{n}$ simply by $G$.

Let $n \geqq 2$ and $G^{n}$ be Gray mapping for $n$ with nodes $\{\xi(1), \ldots, \xi(k)\}$ where also $k-1<\log _{2} n \leqq k$. We define the mapping $C^{n}: W \rightarrow W^{\Sigma}$ recursively where $\Sigma=\{\xi(1), \ldots, \xi(k), \xi(k+1)\}$.

(1) For an integer $m,(0 \leqq m<n)$, we define $C^{n}(m)_{\xi(i)}=G^{n}(m)_{\xi(i)}$ for all $i \leqq k$ and $C^{n}(m)_{\xi(k+1)}=0$.

(2) For an integer $m,(n \leqq m<2 n)$, we define $C^{n}(m)_{\xi(i)}=G^{n}(n-1)_{\xi(i)}+$ $G^{n}(n-1)_{\xi(i)}-G^{n}(n-1-(m-n))_{\xi(i)}=2 G^{n}(n-1)_{\xi(i)}-G^{n}(2 n-m-1)_{\xi(i)}$ for all $i \leqq k$ and $C^{n}(m)_{\xi(k+1)}=1$.

(3) $C^{n}(2 n)_{\xi(i)}=C^{n}(2 n-1)_{\xi(i)}$ for all $i \leqq k$ and $C^{n}(2 n)_{\xi(k+1)}=C^{n}(2 n-1)_{\xi(k+1)}$ $+1=2$.

(4) For an integer $m>2 n$, we define $C^{n}(m)=q C^{n}(2 n)+C^{n}(r)$. Here, $q$ and $r$ be integers determined by $m=2 n q+r, 0 \leqq r<2 n$. For instance, $C^{5}(0)=$ $(0000), C^{5}(1)=(1000), C^{5}(2)=(1100), C^{5}(3)=(2100), C^{5}(4)=(2110), C^{5}(5)=$ (2111) $, C^{5}(6)=(2121), C^{5}(7)=(3121), C^{5}(8)=(3221), C^{5}(9)=(4221), C^{5}(10)=$ $(4222), C^{5}(11)=(5222), C^{5}(12)=(5322), C^{5}(13)=(6322), \ldots$ We call the mapping $C^{n}$ the periodically cumulative Gray mapping for $n$ with nodes $\Sigma$ or simply the periodical Gray mapping for $n$ with nodes $\Sigma$. When $n$ is understood we shall denote $C^{n}$ by $C$.

Lemma 2.12. Let $n$ be an integer greater than one and $k$ be the integer determined by $k-1<\log _{2} n \leqq k$. Let $G$ be the Gray mapping for $n$ with nodes $\Sigma=\{\xi(1), \ldots, \xi(k)\}$ and $C$ be the periodical Gray mapping for $n$ with nodes $\Sigma^{\prime}=\Sigma \cup\{\xi(k+1)\}$. Then we have the following properties:

(1) For any integer $m,(n>m>0), G(m)=G(m-1)+\delta^{\xi(i)}$ for some $i \in K$.

(2) For any integer $m, j,(n>m>j \geqq 0), G(m)_{\xi(i)} \not \equiv G(j)_{\xi(i)}(\bmod 2)$ for some $i \in\{1, \ldots, k\}$.

(3) For any integer $m>0, C(m)=C(m-1)+\delta^{\xi(i)}$ for some $i \in\{1, \ldots$, $k+1\}$.

(4) $C(2 n)_{\xi(i)} \equiv 0(\bmod 2)$ for all $i \in\{1, \ldots, k+1\}$.

(5) For $m \geqq 0$ and $j \geqq 0, C(m)_{\xi(i)} \equiv C(j)_{\xi(i)}(\bmod 2)$ hold for all $i \in\{1, \ldots$, $k+1\}$ if and only if $m \equiv j(\bmod 2 n)$.

(6) For any integer $m$ and $r, C(m+2 n r)-C(2 n r)=C(m)-C(0)=C(m)$.

Proof. Every property follows immediately from the definition. 


\section{$\S 4$. Construction of $\hat{\boldsymbol{q}}$-Extension}

As a consequence of Theorem 2.10 we have only to find a procedure for getting a $\hat{q}$-extension of $(V, h) \mid Q(q)$ for each $q \in\{0,1, \ldots, m\}$ in order to find a digital extension of $(V, h)$. Thus hereafter let $V^{q}, h^{q}, Z^{q}, Q$ and $L^{q}$ denote $V|Q(q), h| Q(q), Z(q) \mid Q(q), Q(q)$ and $L(q) \mid Q(q)$, respectively, where $(V, h)$ is a finite distributive chart, and $Z(q), Q(q)$ and $L(q)$ are the cycle, the set of nodes and the minimum point with respect to the cycle $Z(q)$ for $q$ in 2.1 , respectively, unless explicitly mentioned. The following lemma is an extension of 1.33. Though previously defined terminologies are used, the lemma shows a nature in the lattice point space.

Lemma 2.13. We define a subset $\Phi$ of $V^{q}$ and an integer $e$ as follows:

$$
\begin{aligned}
& \Phi=\left\{M^{q} \in V^{q} ; L^{q} \leqq M^{q} \text { but } L^{q}+Z^{q} \leqq M^{q}\right\} \\
& \alpha=\max \left\{r ;\left(L^{q}+r Z^{q}\right)_{i}^{q} \leqq M_{i}^{q} \text { for some } M^{q} \in \Phi \text { and some } i \in Q\right\} \\
& \beta=\min \left\{s ;\left(L^{q}+s Z^{q}\right)_{i}^{q}>M_{i}^{q} \text { for all } M^{q} \in \Phi \text { and all } i \in Q\right\} \\
& e=\alpha+\beta .
\end{aligned}
$$

Since $\Phi$ is a finite set (2.5), such $\beta$ exists.

Then there exists a node $i \in Q$ such that $A_{i}^{q} \not \equiv B_{i}^{q}\left(\bmod e Z_{i}^{q}\right)$ for two points $A^{q}$ and $B^{q}$ in $V^{q}$ such that $A^{q} \geqq L^{q}, B^{q} \geqq L^{q}$ and $A^{q} \not B^{q}\left(\bmod Z^{q}\right)$. Hereafter such integer $e$ is called the synthetic number of $\left(V^{q}, h^{q}\right)$.

Proof. Let $A^{q}, B^{q}$ be points in $V^{q}$ such that $A^{q} \geqq L^{q}, B^{q} \geqq L^{q}$ and $A^{q} \not \equiv$ $B^{q}\left(\bmod Z^{q}\right)$. Let $M^{q}$ be the minimum point of the $q$-similarity class which has $A^{q}$ as its member, and $N^{q}$ be that of $B^{q}$. Then $M^{q} \geqq L^{q}$ and $N^{q} \geqq L^{q}$ but $M^{q} \geqq$ $L^{q}+Z^{q}$ and $N^{q} \geq L^{q}+Z^{q}$ and $M^{q} \neq N^{q}$ (2.1 and 2.4). Let $r, r(1), s$ and $s(1)$ be the non-negative integers such that

$$
\begin{aligned}
& A^{q}=M^{q}+r Z^{q}+r(1) e Z^{q}, \quad 0 \leqq r<e, \\
& B^{q}=N^{q}+s Z^{q}+s(1) e Z^{q}, \quad 0 \leqq s<e .
\end{aligned}
$$

We only have to show $\left(M^{q}+r Z^{q}\right)_{i} \not \equiv\left(N^{q}+s Z^{q}\right)_{i}\left(\bmod e Z_{i}^{q}\right)$ for some $i \in Q$ in order to prove the lemma. It is no loss of generality that we assume $0 \leqq r \leqq s<e$. Assuming that $\left(M^{q}+r Z^{q}\right)_{i} \equiv\left(N^{q}+s Z^{q}\right)_{i}\left(\bmod e Z_{i}^{q}\right)$ for all $i \in Q, M_{i}^{q}+r Z_{i}^{q}=$ $N_{i}^{q}+s Z_{i}^{q}+k(i) e Z_{i}^{q}$ for integers $k(i)$ (not necessarily non-negative). Since $e Z_{i}^{q} \geqq \beta Z_{i}^{q} \geqq \max \left\{M_{i}^{q}, N_{i}^{q}\right\}-L_{i}^{q} \geqq\left|M_{i}^{q}-N_{i}^{q}\right|=\left|k(i) e Z_{i}^{q}+(s-r) Z_{i}^{q}\right|$ and $0 \leqq s-$ $r<e$, we have $-1 \leqq k(i) \leqq 0$ for all $i \in Q$. If $k(i) \equiv 0$ for all $i \in Q$ or $k(i) \equiv-1$ 
for all $i \in Q$ then $M^{q}+r Z^{q}=N^{q}+s Z^{q}$ or $M^{q}+e Z^{q}=N^{q}+(s-r) Z^{q}$, respectively. In each case $M^{q} \stackrel{q}{\sim} N^{q}$ and therefore $A^{q} \stackrel{q}{\sim} B^{q}$, this contradicts the hypothesis. Hence there exist two nodes $i, j$ such that $M_{i}^{q}=N_{i}^{q}+(s-r) Z_{i}^{q}, M_{j}^{q}+e Z_{j}^{q}=$ $N_{j}^{q}+(s-r) Z_{j}^{q}$. Since $\quad M_{i}^{q}=N_{i}^{q}+(s-r) Z_{i}^{q} \geqq L_{i}^{q}+(s-r) Z_{i}^{q}, s-r \leqq \alpha . \quad$ Then $M_{j}^{q}+e Z_{j}^{q}=N_{j}^{q}+(s-r) Z_{j}^{q} \leqq N_{j}^{q}+\alpha Z_{j}^{q}$ hold and therefore $e Z_{j}^{q} \leqq N_{j}^{q}-M_{j}^{q}+\alpha Z_{j}^{q} \leqq$ $\max \left\{M_{j}^{q}, N_{j}^{q}\right\}-L_{j}^{q}+\alpha Z_{j}^{q}<\beta Z_{j}^{q}+\alpha Z_{j}^{q}=e Z_{j}^{q}$, and this is a contradiction.

We first construct a special type of extension $\left(V^{(i)}, h^{(i)}\right)$ for each $i \in Q$, and later the amalgamation of them is proved to be a $\hat{q}$-extension.

Definition 2.14. Let $e$ be the synthetic number of $\left(V^{q}, h^{q}\right)$ and $i$ be an arbitrarily fixed node in $Q$. Let $G$ be the Gray mapping for $m$ with nodes $\Sigma$ and $C$ be the periodical Gray mapping for $n$ with nodes $\Phi$ where $m=\hat{L}_{i}^{q}+1$, $n=e Z_{i}^{q} / 2$ (since $Z_{i}^{q}$ is even, $n$ is an integer) and $\Sigma=\{\xi(1), \ldots, \xi(f)\}, \Phi=\{\eta(1), \ldots$, $\eta(k), \eta(k+1)\}$ with the integers $f$ and $k$ determined by $f-1<\log _{2} m \leqq f$ and $k-1<\log _{2} n \leqq k$. Here we assume $m \geqq 2, n \geqq 2$ and we shall treat the other cases later. We define a distributive chart $\left(V^{\prime}, h^{\prime}\right)$ with nodes $\{i, \Sigma, \Phi\}$ as follows: $V^{\prime}=\{(t, G(t), 0),(t+1, G(t), 0) ; 0 \leqq t<m\} \cup\{(t, G(m-1), C(t-m+1))$, $(t+1, G(m-1), C(t-m+1)) ; t \geqq m\}$. If $m=1$ and $n \geqq 2$ then we don't use the nodes $\Sigma$ and let

$$
V^{\prime}=\left\{\left(0,0^{\Phi}\right),\left(1,0^{\Phi}\right)\right\} \cup\{(t, C(t-m+1)),(t+1, C(t-m+1)) ; t \geqq 1\} .
$$

If $m \geqq 2$ and $n=1$ then we need not $\Phi$ and let

$$
V^{\prime}=\{(t, G(t)),(t+1, G(t)) ; 0 \leqq t<m\} \cup\{(t, G(m-1)) ; t \geqq m\} .
$$

If both $m=1$ and $n=1$ then $V^{\prime}=V^{q} \mid\{i\}$.

For a point $M^{\prime} \in V^{\prime}$, define $h^{\prime}\left(M^{\prime}\right)_{j}=M_{j}^{\prime}(\bmod 2)$ for all $j \neq i$, and $h^{\prime}\left(M^{\prime}\right)_{i}$ $=h^{q}\left(M^{q}\right)_{i}$ for $M^{q}$ of $V^{q}$ such that $M_{\imath}^{q}=M_{i}^{\prime}$.

The distributivity of $V^{\prime}$ may be easily verified. We call the $\left(V^{\prime}, h^{\prime}\right)$ simple extension of $\left(V^{q}, h^{q}\right) \mid\{i\}$ with nodes $\{i, \Sigma, \Phi\},(\Sigma=\phi$ and/or $\Phi=\phi$ depending on $m=1$ and/or $n=1)$. The amalgamation $\left(V^{\prime}, h^{\prime}\right) \otimes\left(V^{q}, h^{q}\right)$ with nodes $\{Q, \Sigma, \Phi\}$ is called the extension of $\left(V^{q}, h^{q}\right)$ with respect to $i$, written as $\left(V^{(i)}, h^{(i)}\right)$.

Lemma 2.15. Let $\left(V^{(i)}, h^{(i)}\right)=\left(V^{q}, h^{q}\right) \otimes\left(V^{\prime}, h^{\prime}\right)$ be the extension of $\left(V^{q}, h^{q}\right)$ with respect to a node $i \in Q$. Then the following (1) and (2) hold for points $M$ and $N$ of $V^{(i)}$.

(1) If either $M_{i} \leqq \hat{L}_{i}^{q}$ and $M_{i} \neq N_{i}$, or $N_{i} \leqq \hat{L}_{i}^{q}$ and $N_{i} \neq M_{i}$, or $M_{i} \neq N_{i}$ $\left(\bmod e Z_{i}^{q}\right)$, then $h^{(i)}(M) \neq h^{(i)}(N)$. 
(2) If $M|Q \stackrel{\hat{q}}{\sim} N| Q$ and $h^{(i)}(M)=h^{(i)}(N)$, then $M \sim N$.

Proof. (1) If $M_{i} \leqq \hat{L}_{i}^{q}$ and $h^{(i)}(M)=h^{(i)}(N)$, then $M_{i}<m$ and $M_{j} \equiv N_{j}$ $(\bmod 2)$ for all $j \in \Sigma$. Thus we have $M_{i}=N_{i}((2)$ of 2.12). Secondly, suppose that $M_{i} \not \equiv N_{i}\left(\bmod e Z_{i}^{q}\right)$ and $h^{(i)}(M)=h^{(i)}(N)$. Then $M_{i} \geqq m$ and $N_{i} \geqq m$ should hold by the previous result. Let $M \mid \Phi=C(a-m)$ and $N \mid \Phi=C(b-m)$. Since $M_{j} \equiv N_{j}(\bmod 2)$ for all $j \in \Phi, a \equiv b\left(\bmod e Z_{j}^{q}\right)((5)$ of 2.12$)$. Then $M_{i} \equiv N_{i}$ $\left(\bmod e Z_{i}^{q}\right)$ follows from the facts that $M_{i}$ equals either $a$ or $a+1, N_{i}$ equals either $b$ or $b+1$ and $M_{i} \equiv N_{i}(\bmod 2)$. This contradicts the hypothesis.

(2) Suppose that $M|Q \stackrel{\hat{q}}{\sim} N| Q$ and $h^{(i)}(M)=h^{(i)}(N)$ for two points $M, N$ of $V^{(i)}$. Since $h^{(i)}(M)=h^{(i)}(N)$, either $M_{i}=N_{i}$ or $M_{i} \geqq m, N_{i} \geqq m$ and $M_{i} \equiv N_{i}$ $\left(\bmod e Z_{i}^{q}\right)$ holds by (1). In the first place let us assume that $M_{i}=N_{i}$. Then either $M|\{i, \Sigma, \Phi\}=N|\{i, \Sigma, \Phi\}$ or $M|\{i, \Sigma, \Phi\}=N|\{i, \Sigma, \Phi\}+\delta^{j}$ or $N \mid\{i, \Sigma, \Phi\}$ $=M \mid\{i, \Sigma, \Phi\}+\delta^{j}$ for some $j \in\{\Sigma, \Phi\}$ (2.14). However, only $M \mid\{i, \Sigma, \Phi\}$ $=N \mid\{i, \Sigma, \Phi\}$ is possible because $M_{j} \equiv N_{j}(\bmod 2)$ for all $j \in\{\Sigma, \Phi\}$. Since $M|Q \sim N| Q$ in $\left(V^{q}, h^{q}\right)$ and $M|\{i, \Sigma, \Phi\}=N|\{i, \Sigma, \Phi\}, M \sim N$ in $\left(V^{(i)}, h^{(i)}\right)$ (1.26). Secondly, suppose that $M_{i} \geqq m, N_{i} \geqq m, M_{i} \neq N_{i}$ and $M_{i} \equiv N_{i}\left(\bmod e Z_{i}^{q}\right)$. It is no loss of generality that we assume $M_{i}=N_{i}+r e Z_{i}^{q}$ for $r>0$. Since $M_{i} \geqq m$, $N_{i} \geqq m, M_{i}>N_{i}$ and $M_{j} \equiv N_{j}(\bmod 2)$ for all $j \in\{i, \Phi\}$, we have $M|\Sigma=N| \Sigma=$ $G(m-1)$ and $M \mid \Phi=C(p+2 n r)(2.13)$ where we put $N \mid \Phi=C(p)$ (of course $p$ is either $N_{i}-m$ or $\left.N_{i}-m+1\right)$. Let $M^{\prime}, N^{\prime}$ denote $M|\{i, \Sigma, \Phi\}, N|\{i, \Sigma, \Phi\}$, respectively, and we first prove $V_{M^{\prime}}^{\prime}=V_{N^{\prime}}^{\prime}$. Suppose that $L^{\prime} \in V^{\prime}$ and $L^{\prime} \geqq M^{\prime}$. Let $L^{\prime}=\left(L_{i}^{\prime}, G(m), C(q)\right)$, here $q$ equals either $L_{i}^{\prime}$ or $L_{i}^{\prime}-1$. Then $L^{\prime}-M^{\prime}+N^{\prime}$ $=\left(L_{i}^{\prime}-M_{i}^{\prime}+N_{i}^{\prime}, G(m), C(q)-C(p+2 n r)+C(p)\right)$. Since $C(q)-C(p+2 n r)-$ $C(q)=C(q)-C(2 n r)=C(q-2 n r)$ by (2) of 2.12, $q-2 n r=q-\left(M_{i}-N_{i}\right)=q-$ $\left(M_{i}^{\prime}-N_{i}^{\prime}\right)$ and $q=L_{i}^{\prime}$ or $q=L_{i}^{\prime}-1$, we have $L^{\prime}-M^{\prime}+N^{\prime} \in V^{\prime}$. This proves $V_{M^{\prime}}^{\prime} \subset V_{N^{\prime}}^{\prime}$. Similarly we have $V_{N^{\prime}}^{\prime} \subset V_{M^{\prime}}^{\prime}$ and therefore $M^{\prime} \sim N^{\prime}$. Then we have $M \sim N$ in $\left(V^{q}, h^{q}\right) \otimes\left(V^{\prime}, h^{\prime}\right)(1.26)$.

Theorem 2.16. Let $\left(V^{(i)}, h^{(i)}\right)$ be the extension of $\left(V^{q}, h^{q}\right)$ with respect to $i$ with nodes $J^{(i)}=\left\{Q, \Sigma^{(i)}, \Phi^{(i)}\right\},\left(\Sigma^{(i)}=\phi\right.$ and/or $\Phi^{(i)}=\phi$ when $L_{i}^{q}=0$ and/or $e Z_{i}^{q}=2$ respectively), for each $i \in Q$. Then the amalgamation $\left(V^{e}, h^{e}\right)=$ $\otimes\left(V^{(i)}, h^{(i)}\right), i \in Q$, is a $\hat{q}$-extension of $\left(V^{q}, h^{q}\right)$.

Proof. Let $M^{e}$ and $N^{e}$ be points in $V^{e}$ such that $h^{e}\left(M^{e}\right)=h^{e}\left(N^{e}\right)$. If $M^{e}\left|Q \stackrel{\hat{q}}{\sim} N^{e}\right| Q$ does not hold, then either $M^{e} \mid Q \leqq \hat{L}^{q}$ and $M^{e}\left|Q \neq N^{e}\right| Q$ or $N^{e} \mid Q \leqq \hat{L}^{q}$ and $N^{e}\left|Q \neq M^{e}\right| Q$ or $M^{e}\left|Q \$ \hat{L}^{q}, N^{e}\right| Q \ddagger \hat{L}^{q}$ and $M^{e}\left|Q \not \equiv N^{e}\right| Q$ $\left(\bmod Z^{q}\right)$. Assuming $M^{e} \mid Q \leqq \hat{L}^{q}$ and $M^{e}\left|Q \neq N^{e}\right| Q$, there exists a node $i \in Q$ 
such that $M_{i}^{e} \leqq \hat{L}_{i}^{q}$ and $M_{i}^{e} \neq N_{i}^{e}$. Then $h^{(i)}\left(M^{e} \mid J^{(i)}\right) \neq h^{(i)}\left(N^{e} \mid J^{(i)}\right)$ ((1) of 2.15) and this contradicts $h^{e}\left(M^{e}\right)=h^{e}\left(N^{e}\right)$. The case of $N^{e} \mid Q \leqq \hat{L}^{q}$ and $N^{e} \mid Q$ $\neq M^{e} \mid Q$ also leads to a contradiction by the same reason. If $M^{e} \mid Q \$ \hat{L}^{q}$, $N^{e} \mid Q \ddagger \hat{L}^{q}$ and $M^{e}\left|Q \not \equiv N^{e}\right| Q\left(\bmod Z^{q}\right)$, we get that $M^{e}\left|Q \geqq L^{q}, N^{e}\right| Q \geqq L^{q}$ and $M^{e}\left|Q, N^{e}\right| Q$ are not $q$-similar (2.7 and 2.4). Then there exists a node $i \in Q$ such that $M_{i}^{e} \not \equiv N_{i}^{e}\left(\bmod e Z_{i}^{q}\right)(2.13)$ and therefore $h^{(i)}\left(M^{e} \mid J^{(i)}\right) \neq$ $h^{(i)}\left(N^{e} \mid J^{(i)}\right)$ ((1) of 2.15), this contradicts $h^{e}\left(M^{e}\right)=h^{e}\left(N^{e}\right)$. This proves $M^{e}\left|Q \stackrel{\hat{q}}{\sim} N^{e}\right| Q$. Since $h^{(i)}\left(M^{e} \mid J^{(i)}\right)=h^{(i)}\left(N^{e} \mid J^{(i)}\right)$ and $M^{e}\left|Q \stackrel{\hat{q}}{\sim} N^{e}\right| Q$, we have $M^{e}\left|J^{(i)} \sim N^{e}\right| J^{(i)}$ in $\left(V^{(i)}, h^{(i)}\right)((2)$ of 2.15) for all $i \in Q$. Therefore $M^{e} \sim N^{e}(1.26)$.

This completes the construction of a $\hat{q}$-extension of $\left(V^{q}, h^{q}\right)$ for $q \neq 0$. Now we shall construct a $\hat{q}$-extension for $q=0$.

Definition 2.17. When $q=0, V^{q}$ has its maximum point $P$ because $(V, h)$ is finite and $Q(0)$ is not spanned by any cycle. Let $i$ be a node in $Q(0)$ and $G$ be the Gray mapping for $m=P_{i}^{q}$ with nodes $\Sigma=\{\xi(1), \ldots, \xi(k)\}$, where $k$ is the integer determined by $k-1<\log _{2} m \leqq k$. Here we assume $m \geqq 2$, and the other case will be considered later. We define a distributive chart $\left(V^{\prime}, h^{\prime}\right)$ with nodes $\{i, \Sigma\}$ as follows: $V^{\prime}=\{(t, G(t)),(t+1, G(t)) ; 0 \leqq t<m\}$. For a point $M^{\prime} \in V^{\prime}$ we define $h^{\prime}\left(M^{\prime}\right)_{i}=h^{q}\left(M^{q}\right)_{i}$ for $M^{q}$ of $V^{q}$ such that $M_{i}^{q}=M_{i}$, and $h^{\prime}\left(M^{\prime}\right)_{j}=M_{j}^{\prime}$ $(\bmod 2)$ for any $j \in \Sigma$, the amalgamation $\left(V^{\prime}, h^{\prime}\right) \otimes\left(V^{q}, h^{q}\right)$ with nodes $\{Q, \Sigma\}$ is called the extension of $\left(V^{q}, h^{q}\right)$ with respect to $i$, written as $\left(V^{(i)}, h^{(i)}\right)$. When $m=1, \Sigma$ is empty and $\left(V^{q}, h^{q}\right)$ itself is $\left(V^{(i)}, h^{(i)}\right)$.

Lemma 2.18. Let $i$ be a node in $Q(0)$ and $\left(V^{(i)}, h^{(i)}\right)=\left(V^{q}, h^{q}\right) \otimes\left(V^{\prime}, h^{\prime}\right)$ be the extension of $\left(V^{q}, h^{q}\right), q=0$, with respect to the node $i$ which has $\{Q, \Sigma\}$ as its nodes where $\Sigma=\{\xi(1), \ldots, \xi(k)\}$ for the integer $k$ determined by $k-1<$ $\log _{2} m \leqq k$. Here, $m=P_{i}^{q}$ for the maximum point $P^{q}$ of $V^{q}$. Then the following (1) and (2) hold for $M$ and $N$ of $V^{(i)}$.

(1) If $M_{i} \neq N_{i}$, then $h^{(i)}(M) \neq h^{(i)}(N)$.

(2) If $M|Q=N| Q$ and $h^{(i)}(M)=h^{(i)}(N)$ then $M=N$ where $Q$ stands for $Q(0)$.

Proof. Suppose that $M_{i} \neq N_{i}$ and $h^{(i)}(M)=h^{(i)}(N)$. We can assume $M_{i}$ $>N_{i}$. Since $M_{j} \equiv N_{j}(\bmod 2)$ for all $j \in \Sigma, M=\left(M_{i}, G\left(M_{i}\right)\right)$ and $N=\left(M_{i}+1\right.$, $G\left(M_{i}\right)$ ). Then $h^{(i)}(M)_{i} \neq h^{(i)}(N)_{i}$ and this is contradiction. Secondly, suppose that $M|Q=N| Q$ and $h^{(i)}(M)=h^{(i)}(N)$. From the previous result (1), $M_{i}=N_{i}$ 
hold and therefore either $M|\{i, \Sigma\}=N|\{i, \Sigma\}$ or $M|\{i, \Sigma\}=N|\{i, \Sigma\} \pm \delta^{j}$ for some $j \in \Sigma$. However, only $M|\{i, \Sigma\}=N|\{i, \Sigma\}$ is possible because $h^{(i)}(M)$ $=h^{(i)}(N)$. Since $M|Q=N| Q$ and $M|\{i, \Sigma\}=N|\{i, \Sigma\}$, we have $M=N$.

Theorem 2.19. Let $\left(V^{(i)}, h^{(i)}\right)$ be the extension of $\left(V^{q}, h^{q}\right), q=0$, with respect to $i$ with nodes $\left\{Q, \Sigma^{(i)}\right\}$ (if $P_{i}^{q}=1$ then $\Sigma^{(i)}=\phi$ ), for each $i \in Q(0)$. Then the amalgamation $\left(V^{e}, h^{e}\right)=\otimes\left(V^{(i)}, h^{(i)}\right), i \in Q(0)$, is a $\hat{q}$-extension of $\left(V^{q}, h^{q}\right)$.

Proof. Let $M^{e}, N^{e}$ be points in $V^{e}$ such that $h^{e}\left(M^{e}\right)=h^{e}\left(N^{e}\right)$. Since $h^{(i)}\left(M^{e} \mid J^{(i)}\right)=h^{(i)}\left(N^{e} \mid J^{(i)}\right), M_{i}^{e}=N_{i}^{e}$ for all $i \in Q(0)$, hence $M^{e} \mid Q(0)=$ $N^{e} \mid Q(0)$. Then $M^{e}\left|J^{(i)}=N^{e}\right| J^{(i)}$ for all $i \in Q(0)$ ((2) of 2.18) and therefore $M^{e}=N^{e}$.

Lemma 2.20. (When we construct an amalgamation of many state charts, this lemma may save labour). Let $(V, h)$ be a state chart with nodes $J$. Here we assume $J=\{1, \ldots, n\}$ for the sake of brevity. Let $\left(V^{k}, h^{k}\right)$ be a state chart with nodes $J(k)$ such that $J(k) \cap J=\{k\}$ for each $k \in J, J(k) \cap J(m)=\phi$ for any distinct $k$ and $m$ and $\left(V^{k}, h^{k}\right)|\{k\}=(V, h)|\{k\}$. Then $\otimes\left(V^{k}, h^{k}\right), k \in J$, equals to $\left(\cdots\left((V, h) \otimes\left(V^{1}, h^{1}\right)\right) \otimes\left(V^{2}, h^{2}\right) \cdots\right) \otimes\left(V^{n}, h^{n}\right)$.

Proof. Let $J^{e}=J(1) \cup \cdots \cup J(n)$. Then both two amalgamations are equal to the set $\left\{M^{e} \in W^{J^{e}} ; M^{e} \mid J \in V\right.$ and $M^{e} \mid J(k) \in V^{k}$ for all $\left.k \in J\right\}$. The mappings will also be easily proved as equal to each an other.

This completes the specification of the procedure for constructing a digital extension of a binary finite distributive chart.

The sequence of steps in this procedure goes as follows.

(1) Find the cycles $Z(1), \ldots, Z(m)$ of $(V, h)$ and construct the grounds $\left(V^{q}, h^{q}\right)=(V, h) \mid Q(q)$ for all $q \in\{0,1, \ldots, m\}$.

(2) Construct the extension of $\left(V^{(i)}, h^{(i)}\right)$ of $\left(V^{q}, h^{q}\right)$ with respect to $i$ for each $i \in J$.

(3) Make the amalgamation to get a $\hat{q}$-extension $\left(\hat{V}^{q}, \hat{h}^{q}\right)=\otimes\left(V^{(i)}, h^{(i)}\right)$, $i \in Q(q)$, for each $q \in\{0,1, \ldots, m\}$.

(4) Make the amalgamation $\left(V^{e}, h^{e}\right)=\otimes\left((V, h) \otimes\left(\hat{V}^{q}, \hat{h}^{q}\right)\right), q \in\{0,1, \ldots$ $m\}$. Then $\left(V^{e}, h^{e}\right)$ is a digital extension. 


\section{References}

[1] Muller, D. E. and Bartkey, W. S., A theory of asynchronous circuits 1, Univ. of Illinios Digital Computer Laboratory, Report, 75 (1956).

[2] —, A theory of asynchronous circuits 2, ibid, 78 (1957).

[ 3 ] Bartkey, W. S., A theory of asynchronous circuits 3, ibid, 96 (1960).

[4] Noguchi, H., Mathematical theory of asynchronous circuits 1, The Generalized circuits, Mem. School Sci, Engrg. Waseda Univ., 27 (1963), 79-91.

[5] Muller, D. E. and Noguchi, H., Mathematical theory of asynchronous circuits 2, The digital circuits, Bull. Sci. Engrg. Research Laboratory, Waseda Univ., 27 (1964), 85-101.

[6] Hattori, M., Muller, D. E. and Noguchi, H., Mathematical theory of asynchronous circuits 3, The charts, ibid, 28 (1964), 54-75.

[7] Miller, R. E., Switching theory, Vol. 2, Sequential circuits and machines, Wiley, New York, 1965.

[ 8 ] Hattori, M. and Noguchi, H., Synthesis of asynchronous circuits, J. Math. Soc. Japan, 18 (1966), 405-423.

[9] Hattori, M., Existence of digital extensions of semimodular state charts, ibid, 23 (1971), 417-434.

[10] Kimura, I., Space-continuous time-semicontinous theory of speed-independent asynchronous circuits, Information and Control, 22 (1973), 373-393.

[11] Nozaki, A., Stability of asynchronous circuits, unpublished.

[12] Nakamura, T. and Utsunomiya, K., A universal procedure to synthesize semimodular speed independent circuits, Trans. Inst. Elec. Commun. Eng. Japan, 58-D (1975), 609615 (in Japanese).

[13] — On a universal design procedure to realize the semimodular state transition graph, Digital Processes, 3 (1977), 237-257.

[14] Unger, S. H., Asynchronous sequential switching circuits, Wiley, New York, 1969.

[15] Miller, R. E., Some relationships between various models of parallelism and synchronization, IBM Research Report, RC-5074, (1974).

[16] Miller, R. E., Parallel program schemata, in Theoretical computer science, (C.I.M.E.I. Ciclo, Bressanone, 1975), Edizioni Cremonese, Roma, 1975.

[17] Miller, R. E., A comparison of some theoretical models of parallel computation, IEEE Trans. on Comp., C-22 (1973), 710-717.

[18] Nakamura, T., Computation graphs and semimodular circuits, to appear. 
